\title{
Die Galle und Wespe der Cynips superfetationis Gir. Ein Beitrag zur Kenntniss der Cynipiden.
}

Von Josef Paszlavszky, Realschullehrer in Budapest.

Im IX. Bande (1859) der „Verhandl. der k. k. zool.-bot. Gesellschaft in Wien" beschrieb Dr. J. Giraud unter dem oben erwähnten Namen eine Galle, deren Erzeuger ihm unbekannt war. *) Der Erzeuger blieb auch bisher unbekannt; ja selbst die Galle gerieth so ziemlich in Vergessenheit. In Dr. G. Mayr's „Die mitteleuropäischen Eichengallen in Wort und Bild" (1870-1871) ist sie mit einem "?" erwähnt, aber weder abgebildet noch beschrieben. Mir ist es gelungen, sowohl die Galle neu aufzufinden, wie auch die Wespe daraus zu erziehen. Diese Umstände haben mich veranlasst, die kaum gekannten Gallen abzubilden und eine Beschreibung der Wespe zu publiciren. ${ }^{* *}$ )

Ich fand die Gallen am 14. Juli 1882 an einer Qu. pedunculata Ehrh. im Budapester zoologischen Garten; ausserdem brachte mir Professorcandidat J. Vángel aus Peszér zwei Fruchtbecher mit Gallen; schliesslich fand ich im Herbste 1883 ein Exemplar sammt dem Fruchtbecher unter einer Qu. pubescens Willd. am Ofner Hárshegy (Lindenberg).

Einer ausführlichen Beschreibung der äusseren Merkmale der Galle dürfte mich Gira ud's musterhafte Beschreibung derselben wohl entheben. Ich will nur bemerken, dass Gira ud's Beschreibung mehr auf die Gallen von Qu. pubescens Willd. passt, denn diese sind eigentlich "arrondies et couvertes de poils gris, un peu soyeux". - Die Gallen auf der Stieleiche sind citronenförmig und an beiden Enden kegelförmig verlängert. Das Kegelchen an dem oberen freistehenden Ende der Galle ist abgerundet; das am entgegengesetzten Ende befindliche, womit die Galle im Rande des Fruchtbechers sitzt, ist hingegen bedeutend breiter, ein wenig zusammengedrückt und an der Stelle, wo die Galle vom Gewebe des Fruchtbechers sich loslöst, besitzt es eine glatte, dunkel-

*) Dr. J. Giraud, Signalement de quelques espèces nouvelles de Cynipides et de leurs galles. pag. 372.

**) In ungarischer Sprache erschien diese Publication in "Mathematikai és Természettudományi Ėrtesitö" der ungar. Akademie der Wissensch. II. Bd., 2. -3 . Heft, 1883.

„Wiener Entomologische Zeitung“ III. (10. Juni 1884). Heft 5. 
braune, rundliche Oberfläche, ähnlich dem sogenannten Nabel mancher Samen (Bohnen, Kastanien u. s. w.).

Die Farbe der Galle auf dem Baume ist graulich-grün, wie die des Fruchtbechers; die des Kegelchens ist weisslichgelb; dieses ist kahl, während der Körper der Galle mit Seidenhaaren bedeckt ist. Die Galle von Qu. pubescens zeigt in meridianer Richtung einige schwache Rippen und ist, das gelbliche Kegelchen ausgenommen, fast ganz karminroth; ausserdem ist die Behaarung, besonders um das Kegelchen herum, viel reicher und glänzender, als bei den Gallen von Qu. pedunculata.

Auf einem Fruchtbecher sitzt meistens nur eine einzige Galle; jedoch fand ich auch zwei oder auch drei nebeneinander. J. Vángel brachte mir aus Peszér (Pester Comitat) einen grossen Fruchtbecher von $Q u$. pedunculata, an dessen Rande rundherum sechs Gallen sassen.

Die von Gallen besetzten Fruchtbecher bleiben in ihrer Entwicklung nicht immer zurück; dennoch sind sie in den meisten Fällen mehr oder weniger deformirt, indem sie an jenen Stellen, wo die Gallen sitzen, sich verlängern und wegen der wachsenden Galle voluminöser werden. Der eine einzige Galle tragende Fruchtbecher verlängert sich nur nach einer Richtung, der mit zwei Gallen wird nach zwei, der mit drei Gallen nach drei Richtungen u. s. f. deformirt. Die Gallen sitzen mit ihrem unteren Viertel oder Drittel in den Vertiefungen am Rande der Fruchtbecher. Diese Vertiefungen sind $1.5-2 \mathrm{~mm}$ tief, weisslichgelb und am Grunde mit einer dem glatten Nabel der Galle entsprechenden Narbe versehen.

Die Gallen fallen aus dem Fruchtbecher sehr früh heraus und nehmen eine braune Färbung an. Im Jahre 1882 bemerkte ich dieselben auf den Bäumen am 14. Juli, und am 17., also nach drei Tagen, fand ich fast nur mehr leere Fruchtbecher. Diejenigen Gallen, welche nicht abfallen, enthalten regelmässig Parasiten, was auch schon ihre abgeplattete Gestalt verräth.

Die Gallen sind erbsengross, oder, wie sich Giraud ausdrückt, „à peine du volume d'une chevrotine"; $5-6.3 \mathrm{~mm}$ hoch, $4.5-5 \mathrm{~mm}$ breit.

Die Wand einer reifen Galle ist ungefähr $0.5 \mathrm{~mm}$ dick und schliesst eine rundlich geformte, geräumige Larvenkammer ein. Im Durchschnitt lässt sich das übriggebliebene Nahrungs- 
gewebe, das einer Innengalle entsprechende braune und härtere Schutzgewebe und das mehr lockere Gewebe der Aussengalle wahrnehmen, welch' letzteres mit der Innengalle eng verwachsen ist. Eine trockene Galle hat beim Schneiden die Consistenz wie hartes Wachs, was ich dem reichen Oelgehalte der Zellen zuschreibe.

Ein Querschnitt der Gallenwand zeigt unter dem Mikroskope die folgenden Gewebselemente: die Epidermis mit den einzelligen Haaren, die hypodermalen Zellen, eine Art Rindenparenchym, eine parenchymatische Uebergangsschichte, zu welcher sich eine aus verdickten, parenchymartigen Zellen gebildete Schichte gesellt, um das Schutzgewebe der Innengalle zu bilden; endlich das aus dünnwandigen, Oeltropfen enthaltenden Zellen bestehende Nahrungsgewebe.

Die Epidermiszellen zeigen eine starke Cuticulabildung; die einzelnen Zellen mit ihren nach aussen divergirenden Wänden erheben sich nach aussen rund, wie bei manchen Blumenblättern, und verrathen eine starke Neigung. zur Trichombildung. - Die hypodermalen Zellen sind an manchen Stellen nach Art der Korkzellen gebildet; manche sind mit Oel gefüllt. Die dritte Zellenlage kann ich als zerfetztes und ausgetrocknetes Rindenparenchym charakterisiren. Die vierte Lage, welche aus kleinen Parenchymzellen besteht, nenne ich Uebergangsgewebe, denn deren Zellen verdicken sich allmälig, um dann die aus gleichfalls verdickten Zellen bestehende fünfte Lage, das Gewebe der Innengalle zu bilden. In dieser Lage finden sich auch ly sige ne Gäng e (wahrscheinlich Oelgänge), deren längliche Kranzzellen dem inneren Raume mit ihren Längsaxen zugekehrt sind. Die Zellen des Nahrungsgewebes sind sehr dünnwandig. In einem Längsschnitte sind in der Gallenwand auch Spiralgefässe sichtbar. *)

Die graugrüne Farbe der Gallen lässt sie am Rande der Fruchtbecher zwischen dem Laubwerk ebensogut verschwinden, wie später auf dem Boden ihre braune Farbe; ihre Eigenschaft, kurze Zeit nach ihrem Entstehen auf dem Frucht-

*) Die Schnitte wurden von einer ausgetrockneten und in einer Mischung von Alkohol mit Glycerin erweichten Galle genommen; demzufolge kann ich über den Chlorophyll- und Amylumgehalt der Zellen nichts mittheilen. 
becherrande, abzufallen, hat den Zweck, dieselben mit ihren Insassen den Augen der herumjagenden Schmarotzer möglichst $\mathrm{zu}$ entziehen.

Die von mir im Jahre 1882 gesammelten Gallen wurden in einem mit feuchter Erde gefüllten Blumentopfe gehalten. Im April 1883 enthielten die Gallen noch Larven; im September fand ich die Larven in den aufgeschnittenen Gallen todt und vertrocknet. In der Meinung, dass keine Wespen mehr auskriechen dürften, schnitt ich auch die unversehrten Gallen auf und fand in zwei derselben vollkommen ausgebildete Wespen. Es ist höchst wahrscheinlich, dass die Wespen erst im nächsten (1884) Frühjahre, also im Frühjahre des dritten Jahres ausgeflogen wären. Die aus den Gallen geschnittenen Wespen lagen eine Weile schlaftrunken auf dem Rücken und, wenn sie berührt wurden, bewegten sie höchstens die Beine und die Antennen; später krochen sie lebhaft herum und breiteten ihre langen Flügel zum Fluge aus. Bei Berührung stellen sie sich todt. Sie hatten einen sehr schwachen Geruch, dessen Qualität ich nicht bestimmen konnte. Eine der beiden Wespen wurde zur näheren Untersuchung aufgespiesst, die andere aber in einer Eprouvette zwischen das Fenster gestellt, um ihre Lebensdauer und ihre richtige Flugzeit erforschen zu können. - Mit dem Eintritte der kalten Witterung klammerte sie sich an einen Papierstreifen an und blieb regungslos in dieser Stellung; Ende November fand ich sie aber bereits todt; sie hatte also, ausserhalb der Galle, circa zwei Monate gelebt. Es ist nicht ausgeschlossen, dass sie schon im Herbste des zweiten Jahres fliegt.

Bei der näheren Untersuchung stellte sich heraus, dass die superfetationis-Wespe e in Andricus $\mathrm{H}$ art. is t und z u m Subgenus Andricus Hart. (Mayr) gehört. Specifisch ist sie $\operatorname{dem} A$. rhizomae Hart. und A. corticis Hart. am nächsten verwandt. *)

Diagnose:

Andricus superfetationis Gir. (= Cynips superfetationis Gir.). - Das dritte Cubitalfeld ziemlich wasserhell; Fühler ganz braunschwarz; Kopf

*) Vgl. Dr. G. M a yr, Die Genera der gallenbe wo hnenden Cynipiden. Wien 1881. - Die europäischen Arten der gallenbewohnenden Cynipiden. Wien 1882. 
braunroth; Clypeus, Wangen, Interocellargegend und Hinterkopf braunschwarz; Thorax braunroth; Mesonotum mit drei sehr breiten, an der Hinterhälfte zusammenfliessenden schwarzen Längsstreifen, deren mittlerer schon am Vorderrande des Mesonotum, die seitlichen weiter hinten beginnen; Abdomen oben schwarz, unten braunroth; die Seiten des zweiten Segmentes reichlich kurz und seidenartig behaart.

Länge $4 \mathrm{~mm}$. A game Form.

Fliegt wahrscheinlich im Frühlinge des dritten Jahres, oder im Herbste des zweiten Jahres.

Dr. G. Mayr war so gütig, meine Wespe mit denen seiner reichen Sammlung zu vergleichen und gibt noch (in litt.) zur obigen Diagnose Folgendes an: Da s z w eite A bd o m in alsegment oben fast so stark nach hinten verlängertwie bei $A$. Clementinae Gir., viel mehr als bei den sonst sehrnahe stehenden $A$. corticis und rhizomae.

Demzufolge glaube ich die superfetationis-Wespe zwischen A. solitarius Fonsc. und A. corticis Hart. einreihen zu können.

\section{I T E R A T U R.}

Die Zahlen in den Klammern deuten auf die Paginirung der Original-Arbeiten.

\section{Allgemeines.}

Palmén J. A. Ueber paarige Ausführungsgänge der Geschlechtsorgane bei Insecten. Eine morphologische Untersuchung. Mit 5 lithogr. Tafeln. Helsingfors 1884 .

Diese äusserst interessante Publication behandelt in Bezug auf die Insecten die Frage, wie die beiden Formen des Endabschnittes der Geschlechtsgänge, der unpaarige und der paarige, unter sich za vergleichen und morphologisch zu erklären sind. Zur Lösung dieser Frage wurde zum Ausgangspunkte der Untersuchungen und Stadien die Gruppe der Ephemeriden, als hierfür besonders geeignet, gewählt. Die Abhandlung zerfällt in folgende 5 Capitel : 1. Einleitung, in welcher die verschiedenen Forschungsrichtungen in der Entomologie discutirt werden; 2. Berichtef rüherer Autoren überdieGeschlechtsorgane der Ephemeriden; 3. eigene Untersuchungen über die männlichen Geschlechtsorgane der Ephemeriden; 4. eigene Untersuchungen über die weiblichen Geschlechtsorgane der Ephemeriden. In dem letzten (5.) Capitel: Morphologische Ergeb$\mathrm{n}$ isse und Schlüsse zählt der Autor folgende 5 Punkte auf, welche für die Ephemeriden mit wenig Ausnahmen als Regel gelten : „1. Bei beiden Geschlechtern sind die Ausführungsgänge die ganze Strecke hindurch unabhängig von einander 


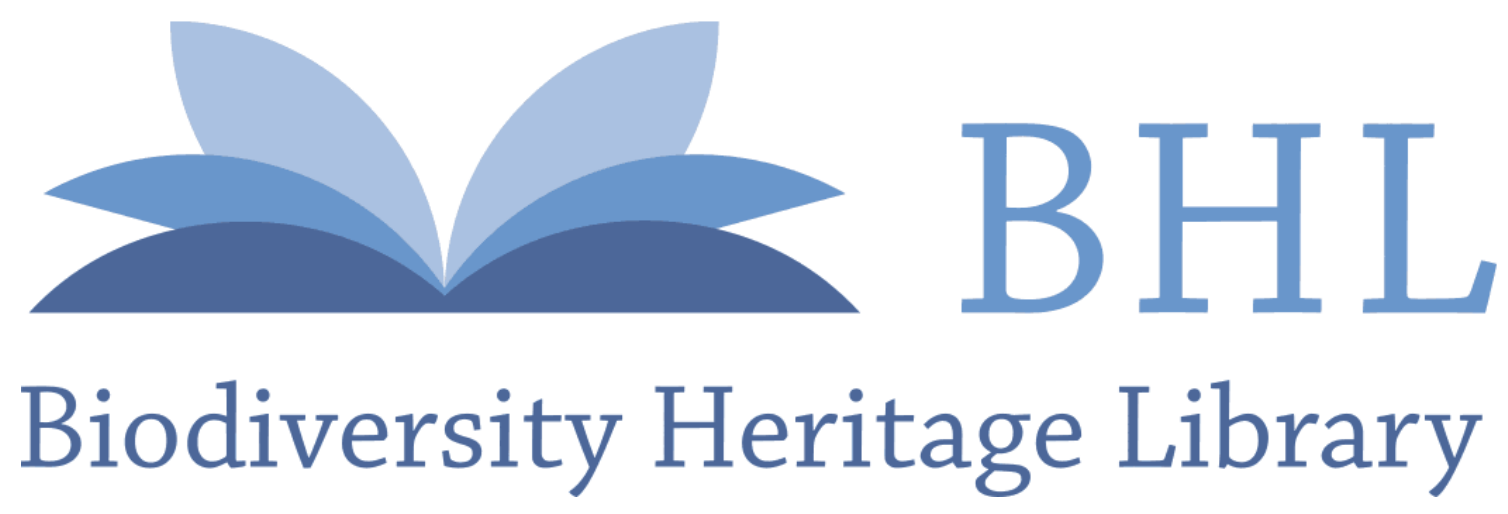

Paszlavszky, Josef. 1884. "Die Galle und Wespe der Cynips superfetationis GIR. Ein Beitrag zur Kenntniss der Cynipiden." Wiener entomologische Zeitung 3, 147-151. https://doi.org/10.5962/bhl.part.13838.

View This Item Online: $\underline{\text { https://www.biodiversitylibrary.org/item/43792 }}$

DOI: https://doi.org/10.5962/bhl.part.13838

Permalink: https://www.biodiversitylibrary.org/partpdf/13838

\section{Holding Institution}

Smithsonian Libraries

\section{Sponsored by}

Smithsonian

\section{Copyright \& Reuse}

Copyright Status: NOT_IN_COPYRIGHT

This document was created from content at the Biodiversity Heritage Library, the world's largest open access digital library for biodiversity literature and archives. Visit BHL at https://www.biodiversitylibrary.org. 